\title{
DESAFÍOS PARA EL CONSUMO DE FRUTAS Y VERDURAS
}

\author{
CHALLENGES FOR THE CONSUMPTION OF FRUITS AND VEGETABLES
}

Mylene Rodríguez-Leyton ${ }^{1, a}$

\begin{abstract}
RESUMEN
Objetivo: Identificar los desafíos a enfrentar para mejorar el bajo consumo de frutas y verduras en Colombia. Métodos: Investigación de tipo documental, descriptivo, exploratorio realizada en los años 2016 y 2017, a partir de la búsqueda, selección y revisión de referencias bibliográficas para analizar y reflexionar acerca de los indicadores de consumo y producción de consumo de frutas y verduras e identificar los desafíos para alcanzar las recomendaciones de la OMS a partir de la evidencia científica. Resultados: Se reafirma el aporte nutricional de las frutas y verduras y sus beneficios para la salud humana, seguida por los indicadores de producción y de consumo como un tema de interés en salud pública; se revisan las normas y lineamientos de política pública que promueven el consumo de una alimentación saludable como estilo de vida y se identifican los mayores desafíos que deben asumir sectores como la industria de alimentos, la gastronomía, el gobierno, las políticas públicas sociales y sectoriales en salud, educación, agricultura, promoción social, comunicación, así como la academia; para definir estrategias efectivas que incrementen los indicadores de consumo de frutas y verduras y permitan alcanzar las metas de un consumo per- cápita mínimo de 5 porciones ó $400 \mathrm{~g}$ diarios propuestas por la Organización Mundial de la Salud (OMS). Conclusión: Diversos países han venido impartiendo lineamientos técnicos para fomentar el consumo de frutas y verduras; sin embargo, es recomendable realizar seguimiento periódico a los indicadores, realizar evaluaciones de resultado e impacto e investigación orientada a identificar las causas de bajo consumo y diseñar estrategias efectivas adecuadas al contexto de las poblaciones.
\end{abstract}

Palabras clave: Frutas; Verduras; Estilo de vida; Alimentación saludable. (fuente: DeCS BIREME)

\begin{abstract}
Objective: Identify the challenges to face in order to improve the low consumption of fruits and vegetables in Colombia. Methods: Documentary, descriptive and exploratory research carried out in 2016 and 2017, based on the search, selection and revision of bibliographic references to analyze and reflect on the indicators of consumption and production of fruit and vegetable consumption and to identify the challenges to reach WHO recommendations based on scientific evidence. Results: the nutritional contribution of fruits and vegetables and their benefits for human health is reaffirmed, followed by production and consumption indicators as a topic of public health interest; the rules and guidelines of public policy that promote the consumption of healthy eating as a lifestyle are reviewed and the main challenges that sectors such as the food industry, gastronomy, government, public social and sectorial policies in health, education, agriculture, social promotion, communication, as well as the academy; to define effective strategies to increase the consumption indicators of fruits and vegetables and to achieve the goals of a minimum per capita consumption of 5 servings or $400 \mathrm{~g}$ per day proposed by the World Health Organization (WHO). Conclusion: Several countries, have been providing technical guidelines to encourage the consumption of fruits and vegetables; however, it is advisable to carry out periodic monitoring of the indicators, perform outcome and impact evaluations and research aimed at identifying the causes of low consumption and designing effective strategies appropriate to the context of the populations.
\end{abstract}

Key words: Fruits; Vegetables; Lifestyle; Healthy diet. (source: MeSH NLM)

'Programa de Nutrición y Dietética, Grupo de Investigación Alimentación y Comportamiento Humano. Universidad Metropolitana de Barranquilla-Colombia.

${ }^{a}$ Nutricionista-Dietista.

Citar como: Mylene Rodríguez-Leyton. Desafíos para el consumo de frutas y verduras. [Artículo de Revisión].2019;19(2):00-00. (Abril 2019). DOI 10.25176/RFMH.v19.n2.2077 


\section{INTRODUCCIÓN}

Alcanzar la recomendación de la Organización Mundial de la Salud (OMS) de un consumo per-cápita mínimo de 5 porciones ó $400 \mathrm{~g}$ de frutas y verduras diarios ${ }^{1}$, como un estilo de vida, es un desafío para las políticas en salud en Colombia y otros países del mundo.

A continuación se presenta la síntesis de una investigación documental orientada a evidenciar los desafíos que enfrenta Colombia para alcanzar el consumo óptimo de frutas y verduras; a partir de la evidencia científica se reconoce su aporte nutricional y los beneficios para la salud humana, seguida por los indicadores de producción y de consumo como un tema de interés en Salud Pública.

Se identifican las normas y lineamientos de política pública para el fomento del consumo de frutas y verduras en Colombia, elemento fundamental de las políticas que promueven la alimentación saludable como estilo de vida y se identifican los mayores desafíos para alcanzar las metas de la OMS.

\section{MÉTODOS}

Se realizó una investigación de tipo documental, descriptivo, exploratorio en los años 2016 y 2017, a partir dela búsqueda, selección y revisión de referencias bibliográficas en bases las bases de datos dialnet, scielo, redalyc, ovid, páginas web de organismos especializados y gubernamentales desde el año 2012 al 2017; con los criterios de búsqueda consumo de frutas y verduras, beneficios su consumo, relación con la salud, composición química, aporte de nutrientes; además se revisaron documentos normativos e informes técnicos. En el caso de los artículos originales de investigación se seleccionaron los publicados en el período establecido; cuando la búsqueda no arrojó información en el período seleccionado, se recurrió a fuentes anteriores al período establecido hasta obtener evidencia científica para analizar y reflexionar acerca de los indicadores de consumo y producción de consumo de frutas y verduras e identificar los desafíos para alcanzar las recomendaciones de la OMS.

\section{RESULTADOS}

El consumo de frutas y verduras guarda una estrecha relación con el estilo de vida, definido por la OMS como una forma general de vida, basada en la interacción entre las condiciones de vida y los patrones individuales de conducta, incorpora un conjunto de valores, normas, actitudes, hábitos y conductas entre ellos la alimentación saludable².

La alimentación saludable permite alcanzar el crecimiento y desarrollo del niño, el mantenimiento de la salud y la actividad del adulto y la supervivencia y bienestar del anciano; favorece y posibilita un buen estado de salud y disminuye el riesgo de enfermedades crónicas relacionadas ${ }^{3}$. Una dieta saludable incorpora variedad de alimentos entre ellos una cantidad de frutas y verduras que contribuyen a elevar el índice la calidad de la dieta y protegen contra el desarrollo de enfermedades.

\section{Características nutricionales de las frutas y verduras}

Las frutas y verduras son fuente significativa de agua y nutrientes como las vitaminas, minerales y fibra, componentes que les confieren propiedades nutritivas y apariencia, textura y color específicas a este grupo de alimentos ${ }^{11}$, tabla 1.

\section{Beneficios para la salud humana del consumo de frutas y verduras}

Existe una amplia evidencia científica acerca de los beneficios para la salud humana del consumo de frutas y verduras, debido a las propiedades derivadas de su composición y combinaciones de nutrientes; así como la presencia de compuestos químicos que intervienen en el metabolismo y liberación de energía en el organismo humano ${ }^{4}$, tabla 2.

\section{Producción de frutas y verduras en Colombia}

Colombia, por su diversidad geográfica, climática y biológica, conforma diferentes ecosistemas, que sumados a la riqueza cultural, su ubicación ecuatorial y la interacción entre estos factores, le permite producir gran variedad de especies vegetales durante todo el año; en un área cercana a los 14 millones de Ha aptas para la producción de alimentos y capital humano para trabajar la tierra. El Censo Nacional Agropecuario en Colombia - 2014, mostró que del área rural dispersa de 111,5 millones de Ha; el 14,6 \% (998.097 Ha) sembrada en frutas y el $4,2 \%$ (233.703) en hortalizas, verduras y legumbres, produciendo $7.746 .235 \mathrm{t}$. de variedad de frutas y $1.433 .022 \mathrm{t}$. de verduras ${ }^{18}$. Ver tabla 3.

\section{Consumo de frutas y verduras}

En Europa el consumo promedio de verduras, legumbres y nueces es de $220 \mathrm{~g}$ por día y el de frutas de $166 \mathrm{~g}$ por día, lo que significa un consumo medio entre frutas y verduras de $386 \mathrm{~g}$ por día ${ }^{19}$.

El estudio prospectivo de epidemiología rural urbana (PURE) en 18 países realizado en 143.305 personas de 18 países según nivel de ingresos mostró una ingesta promedio de 2,19 porciones de verduras con un rango de 2,13-2,25 y de frutas 1,62, con un rango de 1,53 - 1,62. Se observó que los países de bajos ingresos presentan más bajo consumo total de frutas $y$ verduras: 2,14 porciones $(1,93-2,36)$, mientras que en los de ingresos medio-alto fue 4,31 porciones $(4,09$ $-4,53$ ) en los de ingresos medio-bajo 3,17 porciones $(2,99-3,35)^{20}$. 
Tabla 1. Características nutricionales de las frutas y verduras.

\section{NUTRIENTE}

Agua

Fibra

Vitaminas

Minerales

Energía

Lípidos

\section{Carbohidratos}

Proteínas

\section{CONTENIDO EN FRUTAS Y VERDURAS}

Constituyen entre el 60 y $95 \%$ del peso fresco de la porción comestible ${ }^{16}$.

Se presenta en forma por fibra soluble (pectinas) y fibra insoluble (celulosa y hemicelulosa) en proporciones variables según el vegetal, con valor en general entre el 2 y el $9 \%{ }^{17}$.

Forman parte de fitoquímicos o compuestos químicos naturales biológicamente activos que actúan como antioxidantes, es el caso de las vitaminas $A, C$ y E, vitaminas del complejo B como la tiamina, la niacina, la vitamina $\mathrm{B}^{11}$.

El magnesio, el potasio y el zinc se encuentran en cantidades significativas en algunas frutas y verduras ${ }^{15}$.

Las frutas y verduras presentan un contenido calórico relativamente bajo debido a su bajo aporte de grasas y carbohidratos ${ }^{16}$.

El contenido es inferior al 1\%, con excepción de frutas como el coco, el aguacate. Algunas tienen contenido de grasa en forma de ácidos grasos mono y poliinsaturados significativo como el chontaduro $(25,7 \mathrm{gr} / 100 \mathrm{~g})$, el coco $(27,0 \mathrm{gr} / 100 \mathrm{gr})$ y el aguacate $(13,3 \mathrm{~g} / 100 \mathrm{~g})$, La fracción lipídica de las frutas corresponde a acilglicéridos, glicolípidos, fosfolípidos, carotenoides, triterpenoides y ceras $^{11}$.

Se encuentran en forma de fructosa en proporción de 1-8 \% en las frutas siendo mayor en las frutas maduras; en las verduras se encuentra entre el 1-6\%.

La remolacha contiene azúcar $(10,4 \mathrm{~g} / 100 \mathrm{~g})$. Algunas frutas contienen almidones, como la guanábana $(11,9 \mathrm{~g} / 100 \mathrm{~g})$ y del banano $(20,45 \mathrm{~g} / 100 \mathrm{~g})^{16}$.

Las frutas contienen $0,1-1,5 \%$ de compuestos nitrogenados y las hortalizas contienen $1-5 \%$, las proteínas constituyen un $35-75 \%$ en las frutas y el $35-80 \%$ en las hortalizas ${ }^{11}$.

Fuente: Elaboración propia con base en los autores.

Tabla 2. Beneficios para la salud Humana del consumo de frutas y verduras.

\section{BENEFICIO}

Calidad de la dieta

Sobrepeso y Obesidad

Enfermedad isquémica

Niveles de lípidos en sangre

Protección contra adenoma

Disminución de los efectos del estrés oxidativo

Poder antioxidante

Efecto antritrombótico

Protección contra la

hipertensión

\section{EVIDENCIA CIENTÍFICA}

Incrementa el índice de calidad de la dieta ${ }^{4}$.

Disminuyen el tejido graso, controlan el exceso de peso ${ }^{5}$.

Reducen en un $4 \%$ el riesgo de padecer enfermedad isquémica fatal, por cada 80 $\mathrm{g}$ de frutas y verduras adicionales consumidos ${ }^{6}$.

La concentración de triglicéridos y colesterol LDL en sangre es menor en adolescentes que consumen frutas y verduras, comparados con los que tienen baja ingesta ${ }^{7}$.

Presentan efecto protector significativo para adenoma colorectal ${ }^{8}$.

Minimizan el efecto de los radicales libres sobre la alteración las macromoléculas y procesos celulares que originan el cáncer, diabetes y enfermedades reumatoideas ${ }^{9}$.

A mayor contenido de frutas y verduras mayor poder antioxidante total de la alimentación ${ }^{10,11,12}$.

Otorgan efecto antitrombótico y anticoagulante a la dieta ${ }^{13}$.

Ejercen efecto protector contra la hipertensión tanto sistólica como diastólica del consumo de mínimo $400 \mathrm{~g}$ diarios $^{14}$. 
En México, el 34,4 \% de los niños entre 6 y 12 años, cumplía con las recomendaciones diarias del consumo de frutas y verduras, el restante $65,6 \% 21$; mientras que en Argentina el consumo per-cápita de frutas y verduras en la población no alcanza los $200 \mathrm{gr}$ diarios ${ }^{22}$.

Se ha evidenciado que Colombia dispone de una diversidad de productos en el mercado interno de frutas, según la Corporación Colombia Internacional, en el año 2012 se ofrecían 42 tipos de frutas y 30 verduras diferentes en los mercados locales; la regularidad de la oferta depende de decisiones de los agricultores determinadas por el precio, el abastecimiento y de estado sanitario de los cultivos. En cuanto al consumo el Perfil Nacional del consumo de frutas, verduras y hortalizas del año 2012, evidenció que la población colombiana consume en promedio 50 $\mathrm{g}$ diarios de verduras y hortalizas y $94 \mathrm{~g}$ de frutas, que comparados con la recomendación establecida por la OMS, corresponden a un porcentaje de adecuación de
$36 \%$, el mayor porcentaje lo ocupó Bogotá, D.C. con un 44\%; cifra que se encuentra aun significativamente por debajo de lo deseado ${ }^{23}$.

En referencia al consumo diario de frutas y verduras en población colombiana de 5 a 64 años, el 35,3\% de la población no consumió ninguna fruta en su alimentación diaria y $27,9 \%$ no consumió ninguna verdura, comportamiento similar para todos los grupos de población mayor de dos años ${ }^{24}$. Se evidenció mayor preferencia por la frutas, con un consumo por el $66,8 \%$, quienes registraron un consumo diario de tres o más veces al día en el $20,5 \%$. En cuanto a las verduras, el $28,1 \%$ de la población del mismo grupo de edad las consumía diariamente, el 21,7\% una vez al día, el 5,0\% dos veces al día y el 1,4\% tres o más veces al día ${ }^{25}$. En la tabla 4, se observa el porcentaje de personas que consumen las frutas y verduras preferidas por la población colombiana y los gramos per cápita consumidos de cada una de ellas ${ }^{23}$.

Tabla 4. Consumo de frutas y verduras en Colombia.

\begin{tabular}{lcc} 
& PERSONAS QUE CONSUMEN (\%) & grs, PER CÁPITA CONSUMIDOS \\
\hline Limón & Frutas & \\
Banano & 45 & 18 \\
Mango & 99,6 & 15 \\
Guayaba & 123,4 & 15 \\
Tomate de árbol & 95,3 & 14 \\
Mora & 66,2 & 14 \\
Piña & 60,6 & 9 \\
Maracuyá & 77,7 & 8 \\
Naranja & 59,7 & 8 \\
Coco & 154,4 & 7 \\
Aguacate & 51,2 & 5 \\
Papaya & 138,2 & 5 \\
Manzana & 89,4 & 5 \\
& 127,1 & 4 \\
Tomate & Verduras & \\
Cebolla De Bulbo & 33,2 & 59 \\
Zanahoria & 23,21 & 48 \\
Cebolla Larga & 26,54 & 46 \\
Arveja verde & 7,75 & 28 \\
Habichuela & 33,03 & 20 \\
Repollo & 30,45 & 18 \\
Lechuga & 23,09 & 13 \\
Pepino cohombro & 18,16 & 8 \\
Ahuyama & 18,16 & 8 \\
\hline & 12,06 & \\
\hline & & \\
\hline
\end{tabular}

Fuente: Elaboración propia con base en Perfil Nacional de consumo de fruta y verduras. 
Normas, lineamientos y políticas para el fomento del consumo de frutas y verduras en Colombia

En Colombia se acogen y adaptan los lineamientos, normas internacionales y políticas públicas nacionales que legislan la promoción del consumo de frutas y verduras. Ver tabla 5.

Tabla 5. Normas, lineamientos y políticas para el fomento del consumo de frutas y verduras en Colombia.

\section{NORMA, LINEAMIENTO,} POLÍTICA

Declaración de la Cumbre Mundial sobre la Alimentación, FAO 1996.

\section{DATOS DE INTERÉS}

Estrategia mundial sobre régimen alimentario, actividad física y salud, OMS 2004.

Política Nacional de Seguridad Alimentaria y Nutricional, CONPES 113, DNP 2008.

Plan Nacional de Seguridad Alimentaria y Nutricional para Colombia 2012- 2019; Ministerio de salud 2012.

Plan Decenal de Salud Pública de Colombia 2012- 2021, Ministerio de Salud 2013
Reafirmó "el derecho de toda persona a tener acceso a alimentos sanos y nutritivos" $y$ el fomento a la producción de frutas y verduras ${ }^{26}$.

Se incorporan medidas que incluyen el incremento del consumo de frutas y verduras, dada la problemática mundial del bajo consumo ${ }^{27}$.

"Promover hábitos y estilos de vida saludables que permitan mejorar el estado de salud y nutrición de la población y prevenir la aparición de enfermedades asociadas con la dieta" 28 .

"Contribuir al mejoramiento de las condiciones de salud y nutrición del país mediante el incremento en la producción y el consumo de frutas y verduras inocuas" 29 .

Contempla la dimensión de modos de vida y condiciones no transmisibles y establece la meta de "incrementar el consumo diario de frutas y verdura en la población en general" 30 .
Ley 1355 de 2009: ley de obesidad en Colombia, Congreso de Colombia 2009.

Lineamiento técnico nacional para la promoción de frutas y verduras, Ministerio de salud y protección Social de Colombia y FAO 2012.
Establece la promoción del consumo de las frutas y verduras en las Instituciones Educativas públicas y privadas y la garantía de su disponibilidad ${ }^{31}$.

Es un marco de referencia para la definición e implementación de estrategias que fomenten condiciones de disponibilidad y abastecimiento favorables para el consumo de frutas, verduras y hortalizas a nivel nacional, regional y local; establece acciones de regulación, investigación, información, comunicación, educación y movilización social ${ }^{32}$.
Guía alimentaria para la población colombiana, ICBF 2015.
Recomienda incluir frutas enteras y verduras preferiblemente crudas, en cada una de las comidas, para mejorar la digestión, piel y el peso corporal ${ }^{33}$.

Fuente: Rodríguez LM, Sánchez ML. Consumo de frutas y verduras beneficios y retos. Alimentos Hoy. 2017; 25(42): 30-55²4.

Desafíos para el consumo de frutas y verduras en Colombia

La existencia de marcos normativos y regulatorios demuestra que en Colombia las autoridades gubernamentales han venido asumiendo importantes retos para el logro de las metas sobre el consumo de frutas y verduras pero diferentes sectores y actores aún deben enfrentar grandes desafíos ${ }^{34}$. 
Tabla 6. Desafíos para lograr el consumo de frutas y verduras.

ACTORES

DESAFIOS
Industria y la Gastronomía políticas públicas

Salud, educación, agricultura, promoción social, comunicación
El mayor contenido de fibra ${ }^{35-36}$ le otorga un perfil de sabor de baja palatabilidad especialmente a las verduras, principalmente por ser bajos en grasas30; por lo tanto se debe promover la formulación de alimentos de buen sabor y con un alto contenido de nutrientes, que sean sensorialmente atractivos para la población contribuyendo a mejorar su ingesta sin deteriorar su composición nutricional.

La industria tiene el desafío de equilibrar la oferta de alimentos no saludables como bebidas azucaradas y alimentos procesados que son utilizados como sustitutos de las frutas y verduras poniendo al alcance de los consumidores alternativas atractivas y con alta una relación costo- beneficio ${ }^{37}$.

Evaluar los resultados de las normas, políticas y estrategias para promover el consumo de frutas y verduras, verificar su costo- efectividad y diseñar políticas aplicables en los diferentes eslabones de la cadena agroalimentaria, que eviten sus pérdidas y desperdicios.

Transformar los comportamientos relacionados con el consumo de frutas y verduras en las personas ${ }^{37-38-40-41}$.

Generar entornos saludables en mercados, instituciones educativas de todos los niveles y ambientes laborales ${ }^{42-43-44-45-46}$.

Intervenciones educativas con la participación y el empoderamiento comunitario para la promoción de la alimentación saludable han mostrado efectividad, acompañadas de estrategias para incrementar la disponibilidad de frutas y verduras ${ }^{47-48-49-50}$.

Academia

Aportar evidencia científica para el desarrollo e innovación de productos que incluyan como ingrediente frutas y verduras; realizar estudios analíticos y experimentales para probar estrategias de fomento de consumo de frutas y verduras.

Fuente: Elaboración propia.

\section{CONCLUSIÓN}

La evidencia científica refleja ampliamente que las frutas y las verduras constituyen un grupo de alimentos saludables para el ser humano, por su alto contenido de nutrientes;

Colombia cuenta con producción y disponibilidad significativa, pero selección poco variada que se reflejan en baja preferencia y consumo de frutas y verduras como se demostró en el estudio del perfil de consumo. Los indicadores de bajo consumo de frutas y verduras son coherentes con la situación de salud y la doble carga de la malnutrición del país ${ }^{45,46}$.

Colombia ha venido impartiendo lineamientos técnicos como una forma de asumir el reto de mejorar los indicadores de consumo de frutas y verduras; sin embargo, estos marcos normativos no son suficientes para garantizar la efectividad de las intervenciones, las cuales deben ser evaluadas, fortalecidas, ajustadas y/o renovadas.

Es necesario diseñar estrategias efectivas adecuadas al contexto social, cultural y económico de las poblaciones y realizar seguimiento periódico a los indicadores, evaluaciones de resultado e impacto y desarrollar investigación orientada a identificar las causas de bajo consumo, identificar e intervenir las barreras y restricciones, considerando la alta oferta de alimentos que se consumen como sustitutos.

Contribuciones de autoría: El autor participó en la génesis de la idea, diseño de proyecto, recolección e interpretación de datos, preparación del manuscrito y aprobación de la versión final del artículo.

Financiamiento: Autofinanciado.

Conflicto de interés: El autor declara no tener conflicto de interés.

Recibido: 18 de noviembre de 2018

Aprobado: 22 de enero de 2019

Correspondencia: Mgr. Mylene Rodríguez Leyton.

Dirección: Calle $76 N^{\circ}$ 42- 78. Universidad Metropolitana de Barranquilla. Barranquilla - Colombia.

Celular: +5753137971655

Correo:myrodriguez@unimetro.edu.co,mylenerod@gmail.com. 


\section{REFERENCIAS BIBLIOGRÁFICAS}

1. Organización Mundial de la salud. Estrategia mundial sobre régimen alimentario, actividad física y salud. [Internet] Ginebra (Suiza): OMS, 2004 [citado 10 de octubre de 2018]. Recuperado a partir de: http://who.int/ dietphysicalactivity/strategy/ eb11344/

2. World Health Organization. Life styles and Health. Rv.Social Science and Medicine, 1986. 22 (2): 117-124

3. Calañas-Continente AJ., Bellido D. Bases científicas de una alimentación saludable. Rev Med Univ Navarra. 2006; 50(4): 7-14

4. Gil A, Martinez de Victoria E and Olza. Indicators for the evaluation of diet quality. Nutr Hosp. 2015; 31(3):128-44.

5. Arribas H, Battistini T, Rodriguez M, Ortiz A. Asociación entre obesidad y consumo de frutas y verduras: un estudio de base poblacional en Perú. Rev. chil. Nutr. 2015; 42(3): 241-7.

6. Crowe F, Roddam A, Key T, Appleby P, Overvad K, Norat T. Fruit and vegetable intake and mortality from ischaemic heart disease: results from the European Prospective Investigation into Cancer and Nutrition (EPIC)Heart study. European Heart Journal. 2011; 32(10): 1235-43.

7. Parra B, Manjarrés L, Velásquez C, Agudelo G, Estrada A, Uscátegui R et al. Perfil lipídico y consumo de frutas y verduras en un grupo de jóvenes de 10 a 19 años, según el índice de masa corporal. Rev Col Card. 2015 22(2): 72-80.

8. Ben Q, Zhong J, Liu J, Wang L, Sun y, Yv L, et al. Association Between Consumption of Fruits and Vegetables and Risk of Colorectal Adenoma: A PRISMA-Compliant Meta-Analysis of Observational Studies. Medicine [Internet]. 2015 [citado 10 de octubre de 2018]; 94(42): 1- 12 Recuperado a partir de: https://ncbi.nlm.nih.gov/pmc/articles/PMC4620815/.

9. Araya L, Clavijo R, Herrera C. Capacidad antioxidante de frutas y verduras cultivados en Chile. ALAN. 2006; 56 (4), 361-5.

10. Messina D, Pérez R, Soto C, Uvilla A, López J, López C. El consumo elevado de licopeno sumado a una ingestión reducida de carnes rojas aumenta el poder antioxidante total. ALAN. 2012; 62 (1) 6-14.

11. Morillas J., Delgado J. Análisis nutricional de alimentos vegetales con diferentes orígenes: Evaluación de capacidad antioxidante y compuestos fenólicos totales. Revista nutrición clínica y dietética hospitalaria. 2012; 32 (2): 8-20.

12. Cruz M, González J, Sánchez P. Propiedades funcionales y beneficios para la salud del licopeno. Nutr. Hosp. 2013; 28(1): 6-15.

13. Torres C, Guzmán L, Moore R, Palomo G . Efecto antitrombótico, una característica poco conocida de las frutas y hortalizas. Rev. chil. nutr. 2008; 35 (1): 10-7.

14. Pienovi L, Lara $M$, Bustos $P$, Amigo H. Consumo de frutas, verduras $y$ presión arterial. Un estudio poblacional. ALAN. 2015; 65 (1): 21- 6.

15. Yahia EM, Irigoyen LE. Frutas y hortalizas en la nutrición humana. Revista Horticultura 161. 2002.

16. Instituto Colombiano de Bienestar Familiar, ICBF.Tabla de Composición de Alimentos Colombianos. Bogotá; 2015.

17. Escudero E, González P. La fibra dietética. Nutrición Hospitalaria 2006; 21(Supl. 2): 61-72.

18. Departamento Administrativo Nacional de Estadística. Tercer Censo Nacional Agropecuario tomo 2: Resultados. [Internet]. Bogotá (Colombia): DANE, 2016 [citado 10 de octubre de 2018]. Recuperado a partir de: https://dane.gov.co/.

19. Revistas Enfasis Alimentación. Consumo de frutas y verduras en Europa: ¿Toman suficiente los europeos? [Internet].México: 2012 [citado el 10 de octubre de 2018]. Recuperado a partir de: http://.alimentacion. enfasis.com/articulos/
20. Miller V, Yusuf S, Chow CK, Dehghan M, Corsi DJ, Lock K, et al. Availability, affordability, and consumption of fruits and vegetables in 18 countries across income levels: findings from the Prospective Urban Rural Epidemiology (PURE) study. The Lancet Global Health 2016; 4 (10): e695 - e703.

21. Jiménez A, Gaona E, Mejía F, Gómez L, Méndez H y Flores $M$. Consumption of fruits and vegetables and health status of Mexican children from the National Health and Nutrition Survey. 2014; 56 Supl. 2: 103-12.

22. Montián GN y Balaban DM. Consumo de frutas y hortalizas en la actualidad. [Internet]. Rosario (Argentina). Universidad Nacional del Rosario; 2014; [citado 10 de octubre de 2018] 39:30-32. Recuperado a partir de: http://.fcagr.unr.edu.ar/?p=5327.

23. Instituto Colombiano de Bienestar Familiar. Encuesta Nacional de la Situación Nutricional en Colombia - ENSIN 2005-.-.[nternet]. Bogotá (Colombia):ICBF; 2006; 466 p. [citado 10 de octubre de 2018]. Recuperado a partir de: http://www.icbf.gov.co

24. Instituto Colombiano de Bienestar Familiar. Encuesta Nacional de la Situación Nutricional en Colombia - ENSIN 2010-.[Internet]. Bogotá (Colombia):ICBF; 2010;513 p. [citado 10 de octubre de 2018]. Recuperado a partir de: http://www.icbf.gov.co

25. Ministerio de salud y protección social - Organización de las Naciones Unidas la agricultura y la alimentación. Perfil Nacional de consumo de Frutas y Veduras. Bogotá: FAO, Febrero de 2013.

26. Food and Agriculture Organization of the United Nations. Informe de la Cumbre Mundial sobre la Alimentación. [Internet]. Roma (Italia): FAO; 1996 [citado 10 de octubre de 2018]. Recuperado a partir de: http://fao. org/docrep/003/w3548s/

27. World Health Organization. WHO. Estrategia mundial sobre régimen alimentario, actividad física y salud, Fomento del consumo mundial de frutas y verduras. [Internet] Ginebra (Suiza): [citado 10 de octubre de 2018]. Recuperado a partir de: http://who.int/dietphysicalactivity/fruit/es/.

28. Departamento Nacional de Planeación. Política Nacional de Seguridad Alimentaria y Nutricional, Documento Conpes Social 113 de Marzo 31 de 2008.

29. Ministerio de Salud y Protección Social, Gobierno de Colombia. Plan Nacional de Seguridad Alimentaria y Nutricional (PNSAN) 2012-2019. Bogotá D.C.; Diciembre 17 de 2012.

30. Ministerio de Salud y Protección Social, Gobierno de Colombia. Plan Decenal de Salud Pública, 2012-2021. Bogotá D.C., Cundinamarca; Marzo 13 de 2013.

31. Congreso de la República de Colombia. Ley 1355 de 2009: por medio de la cual se define la obesidad y las enfermedades crónicas no transmisibles asociadas a esta como una prioridad de salud pública y se adoptan medidas para su control, atención y prevención. Bogotá D.C.; Diario Oficial 47502; Octubre 14 de 2009 [citado 10 de octubre de 2018]. Recuperado a partir de: http://docs.colombia.justia.com/nacionales/ leyes/ley-1355-de-2009

32. Ministerio de Salud y Protección Social, Gobierno de Colombia y Organización de las Naciones Unidas para la Agricultura y la Alimentación, FAO, Bogotá; acuerdo No. 389 de 2012: Lineamiento técnico nacional para la promoción de frutas y verduras. [citado 10 de octubre de 2018]. Recuperado a partir de: http://osancolombia.gov.co/doc/

33. Instituto Colombiano de Bienestar Familiar. Documento Técnico Guías Alimentarias basadas en Alimentos para la población Colombiana Mayor de 2 años. Bogotá D.C., Noviembre de 2015.

34. Rodríguez LM, Sánchez ML. Consumo de frutas y verduras beneficios y retos. Alimentos Hoy. 2017; 25(42): 30-55. 
35. Ham E, kim J. Evaluation of fruit intake and its relation to body mass index of adolescents. Clinical nutrition Research. 2014; 3(2): 126-33.

36. Almeida AS, Aguilar AS, Hervert HD. La fibra y sus beneficios a la salud. An Venez Nutr. 27(1): 73-76. 2014.

37. Cobiac L, Theo V, Veerman L. Cost-effectiveness of interventions to promote fruit and vegetable consumption. Plos One. 5(8): p. 1- 8. 2010.

38. Herrera S, Panader A, Cárdenas LM, Agudelo N. Promoción de una alimentación saludable: experiencia en Tunja, Colombia. Av Enferm. 2012; 30(1): 55-63.

39. Gamboa D, López B, Prada G, Franco C, Landínez N. Factores asociados al consumo de frutas y verduras en Bucaramanga, Colombia. ALAN. 2010; 60(3): 247-53

40. Arboleda LM, Duque M, Urrea J. Significados del consumo de frutas y hortalizas. Saúde Soc. São Paulo. 2013; 22(4): 1247-56.

41. Araneda F, Ruiz N, Vallejos V, Oliva M. Consumo de frutas y verduras por escolares adolescentes de la ciudad de Chillán. Chile. Rev chil nutr. 2015; 42(3): 248-53.

42. Bigio RS, Verly E, De Castro M, Galvão C, Fisberg RM, Lobo D. Determinants of fruit and vegetable intake in adolescents using quantile regression. Rev Saúde Pública 2011;45(3): 1-8

43. Cleyton A, Marciniak A, Dos Santos, L, Barretta C, Nesello N, Nottar $\mathrm{L} \hat{A}$. Factors associated with consumptionof fruits and vegetables by teenagers in Penha, Brazil. ActaScientiarum. Health Sciences. 2015; 37(2): 197-203.
44. Díaz-Beltrán MP. Factores influyentes en el comportamiento alimentario infantil. Rev Fac Med. 2014; 62(2): 237-45.

45. Chaffee B. Fatores nos primeiros anos de vida que influenciam o consumo de frutas e verduras entre criancas. J Pediatr. 2014; 90(5): 437-39.

46. Restrepo B, Urango M, Deossa R. Consumo de vegetales y factores relacionados en estudiantes universitarios de la ciudad de Medellín, Colombia. Perspectivas en Nutrición Humana. 2013; 15(2): 171-83.

47. Meléndez L, Olivares S, Lera M. Mediano S. Etapas del cambio, motivaciones y barreras relacionadas con el consumo de frutas y verduras y la actividad física en madres de preescolares atendidas en centros de atención primaria de salud. Rev Chi Nut. 2011; 38(4): 466-75.

48. Vitolo R y Valmórbida J. Factors associated with low consumption of fruits and vegetables by preschoolers of low socio-economic. J Pediatr. 2014; 90(5): 464- 71

49. Costa B, Oliveira D and Lopes A. Food environment of fruits and vegetables in the territory of the Health Academy Program. Cad. Saúde Pública. 2015; 13 (suppl.1): 159-69.

50. González C, Zacarías I, Domper, Fonseca M. Lera M. Vio del R. F. Evaluación de un programa de entrega de frutas con educación nutricional en escuelas públicas rurales de la Región Metropolitana, Chile. Rev Chil Nutr. 2014; 41(3): p. 228-35.

\section{latindex}

http://www.latindex.org/latindex/ficha?folio=14280

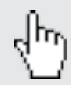

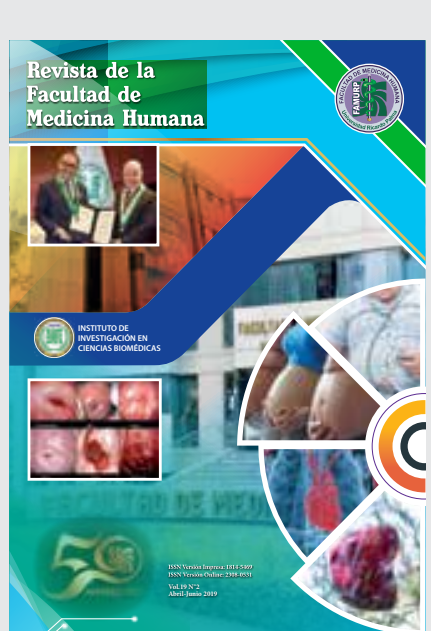

